\title{
Magnetic headspace adsorptive extraction of chlorobenzenes prior to thermal desorption gas chromatography-mass spectrometry
}

Lorena Vidal ${ }^{\mathrm{a}, *}$

lorena.vidal@ua.es

Mazaher Ahmadi

Elen

Tayyebeh

Antonio Canals ${ }^{\mathrm{a}, * *}$

a.canals@ua.es

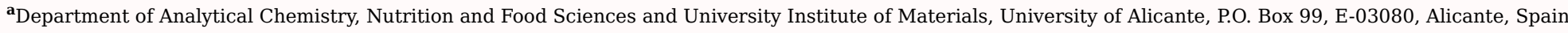

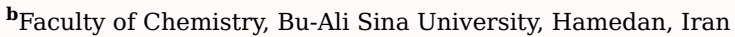

${ }^{*}$ Corresponding author.

${ }^{* *}$ Corresponding author.

Abstract

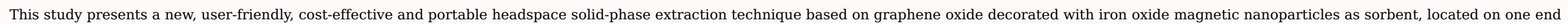

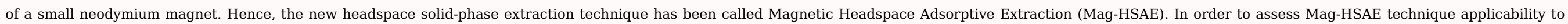

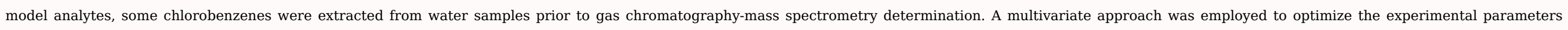

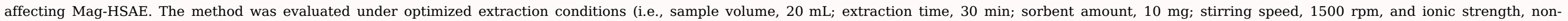

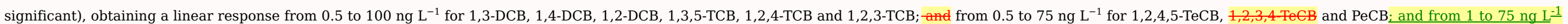

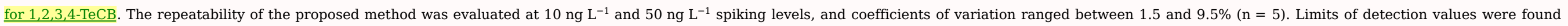

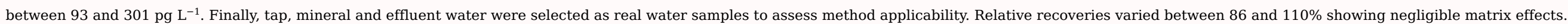

Keywords: Solid-phase microextraction; Chlorobenzenes; Magnetic graphene oxide; Magnetic headspace adsorptive extraction; Gas chromatography-mass spectrometry; Water samples

\section{Introduction}

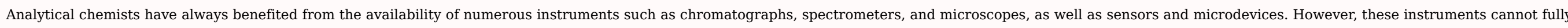

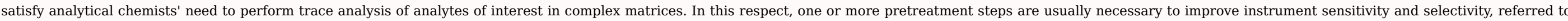

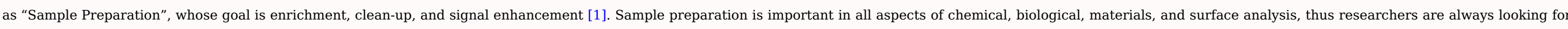

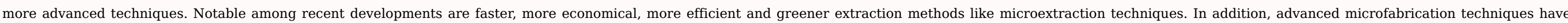
resulted in the development of miniaturized chemical analysis systems, including microscale sample preparation on a chip [2].

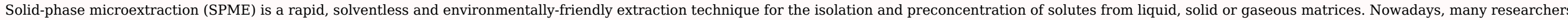

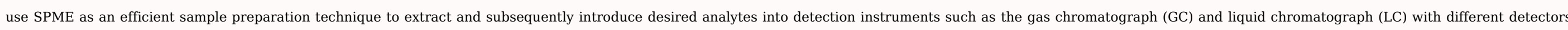

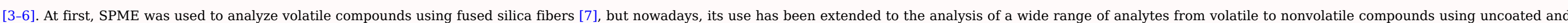




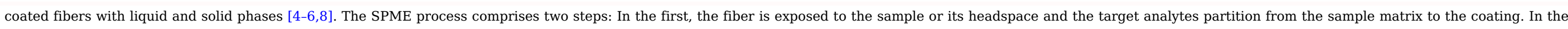

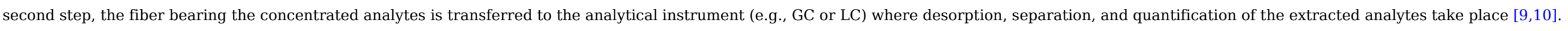

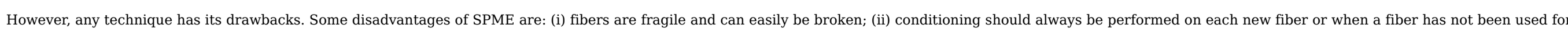

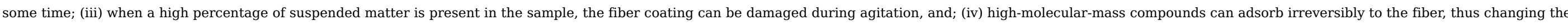

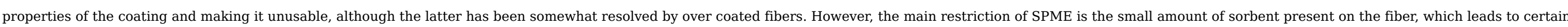

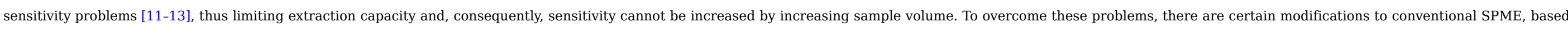

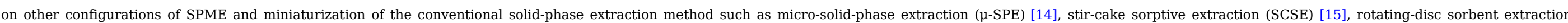

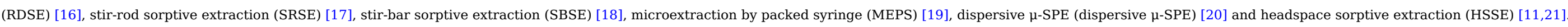

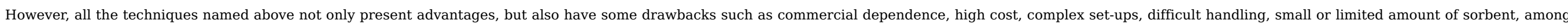

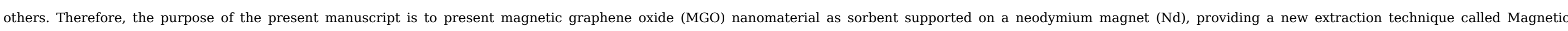
Headspace Adsorptive Extraction (Mag-HSAE), which overcomes the drawbacks of most of the extraction techniques described above.

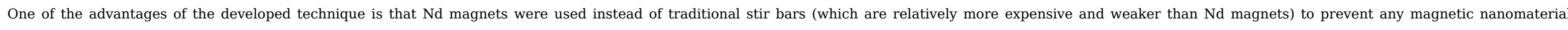

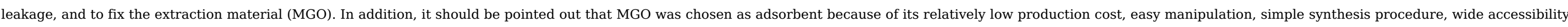

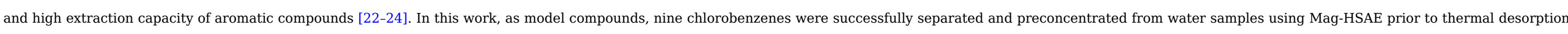

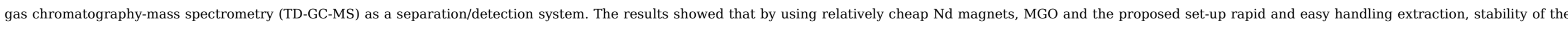

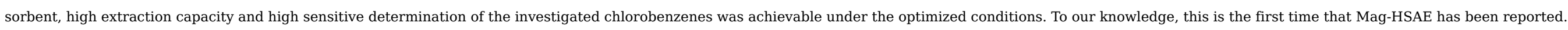

\section{Experimental}

\subsection{Chemicals and real samples}

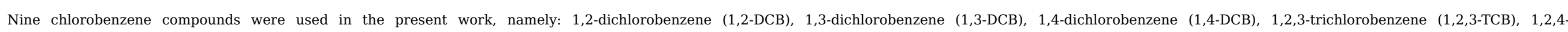

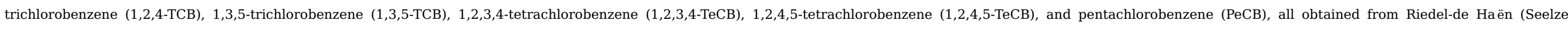

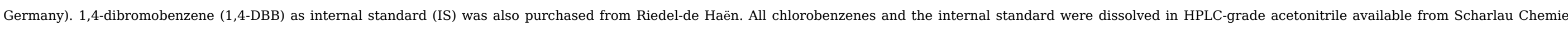

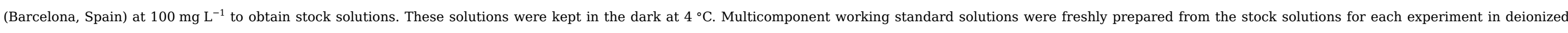
water (resistivity of $18.2 \mathrm{M} \Omega \mathrm{cm}$ at $25^{\circ} \mathrm{C}$ ), supplied using a water purification system (Milli-Q Biocel A10) from Millipore (Billerica, MA, USA).

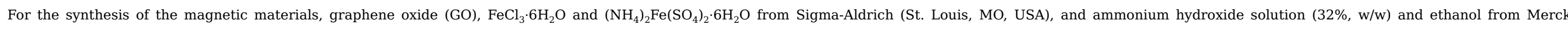

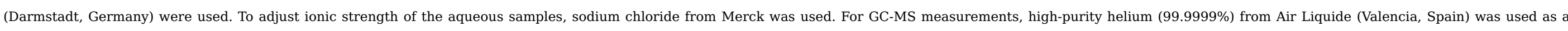
carrier, for both thermal desorption and GC processes. Liquid nitrogen, also from Air Liquide, was used as a coolant for the cooled GC injection system.

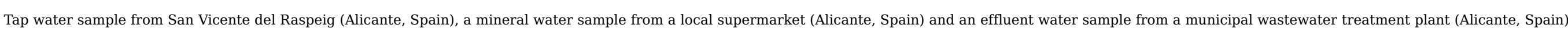

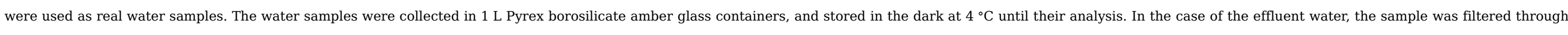
syringe filters (Acrodisc ${ }^{\circledR}$, pore size $0.45 \mu \mathrm{m}$ ) before extraction.

\subsection{Apparatus and instruments}

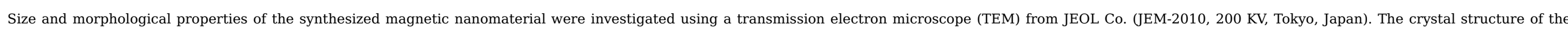

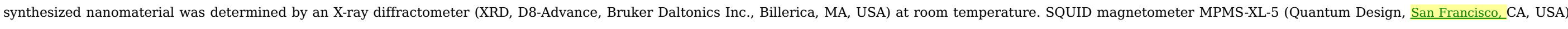
was used for magnetic characterization. A $35 \mathrm{kHz}$ universal ultrasonic cleaner water bath (Elma, Singen, Germany) was used for the nanomaterial synthesis.

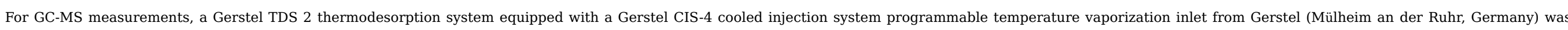




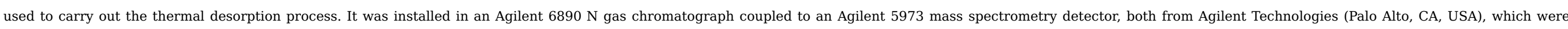

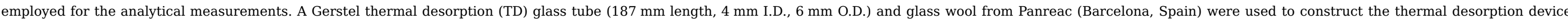
(Fig. 1), which enabled the desorption of the compounds from the magnetic nanomaterial, while preventing them from entering the GC system.

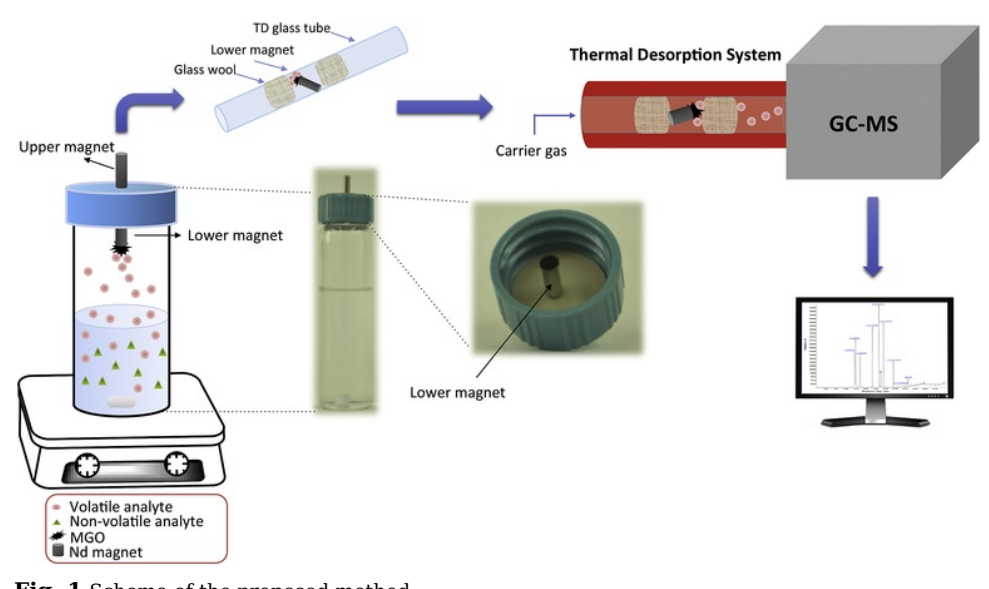

Fig. 1 Scheme of the proposed method.

\section{alt-text: Fig. 1}

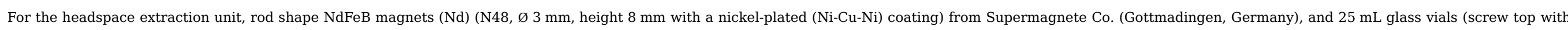
solid green Melamine cap with PTFE liner) from Supelco (Bellefonte, PA, USA) were used (Fig. 1). Due to the strength and dimensions of the Nd magnet used non-special precaution was required.

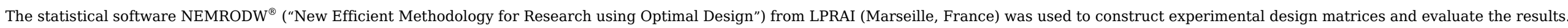

\subsection{Synthesis of magnetic graphene oxide}

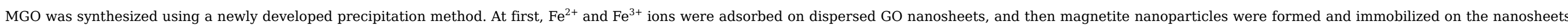

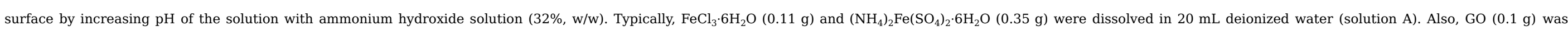

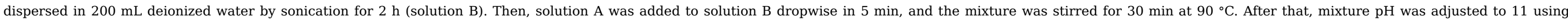

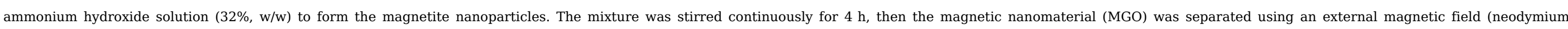
magnet), washed using deionized water and ethanol (3 consecutive times), and dried at $200^{\circ} \mathrm{C}$ for $2 \mathrm{~h}$.

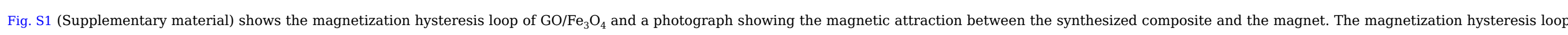
of $\mathrm{GO} / \mathrm{Fe}_{3} \mathrm{O}_{4}$ was S-like curve indicating that the composite was superparamagnetic material. The specific magnetization saturation $\left(\mathrm{M}_{\mathrm{s}}\right)$ was 38.5 emu $\mathrm{g}^{-1}$.

\subsection{TD-GC-MS conditions}

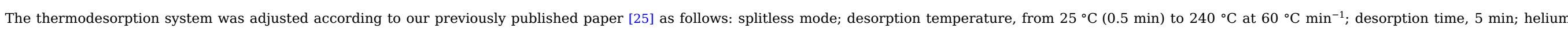

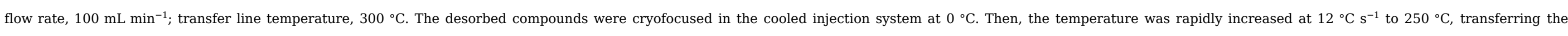

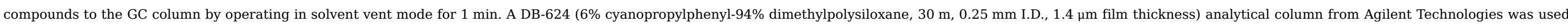
with the following oven temperature program: from $70^{\circ} \mathrm{C}(2 \mathrm{~min})$ at $5{ }^{\circ} \mathrm{C} \mathrm{min}^{-1}$ to $130^{\circ} \mathrm{C}$, and then at $10^{\circ} \mathrm{C} \mathrm{min}^{-1}$ to $240{ }^{\circ} \mathrm{C}(5 \mathrm{~min})$.

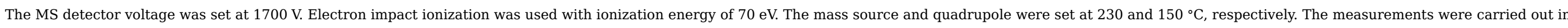

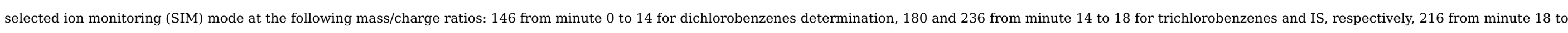




\subsection{Mag-HSAE-TD-GC-MS procedure}

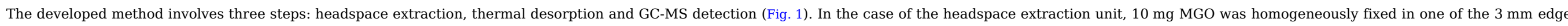

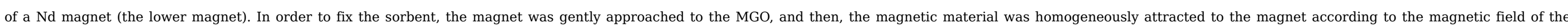

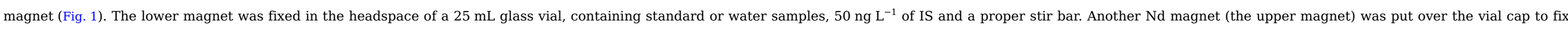

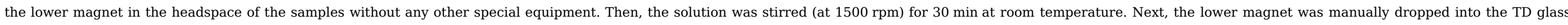

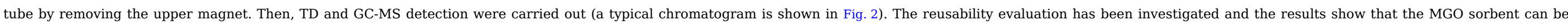

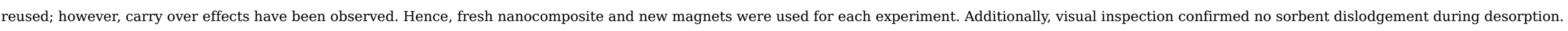

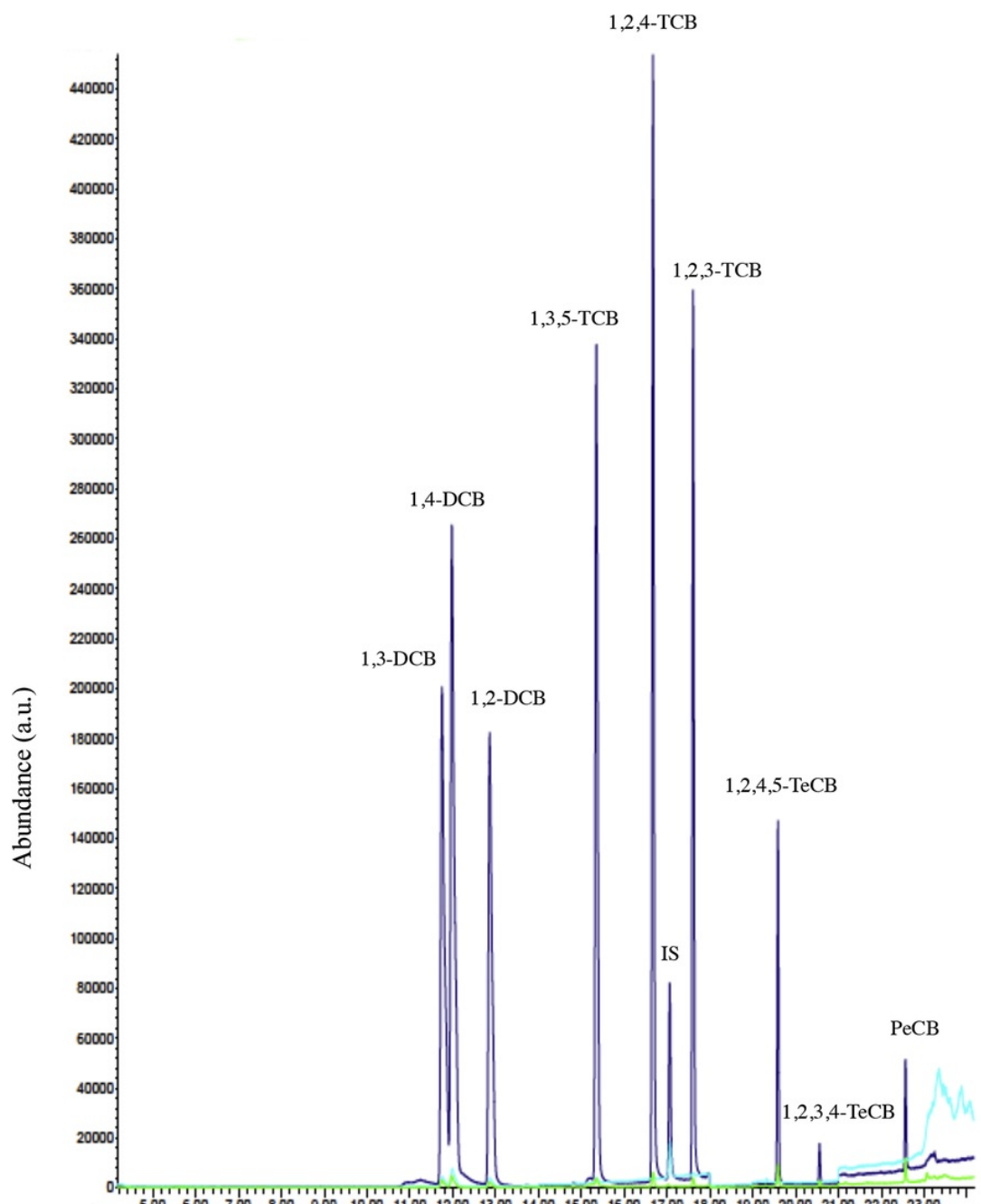

5.00 6.00 P.

Time (min) 


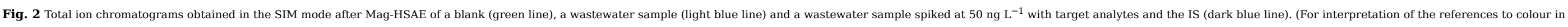
this figure legend, the reader is referred to the web version of this article.)

alt-text: Fig. 2

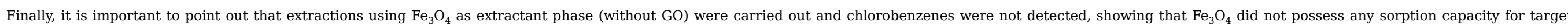
analytes.

\section{Results and discussion}

\subsection{Characterization of the synthesized magnetic nanomaterial}

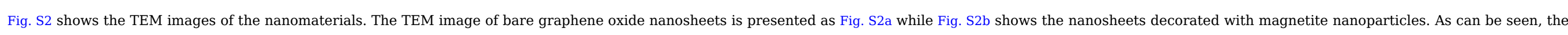
nanosheets are almost uniformly modified by the magnetic nanoparticles with an average size of $14 \pm 3 \mathrm{~nm}$.

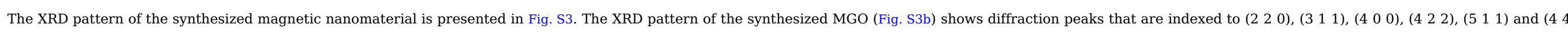

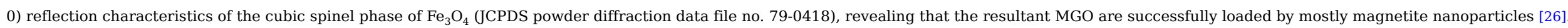

\subsection{Mag-HSAE optimization}

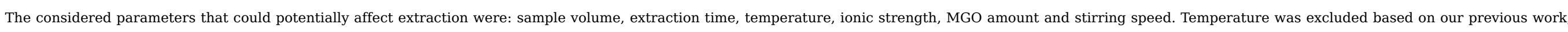

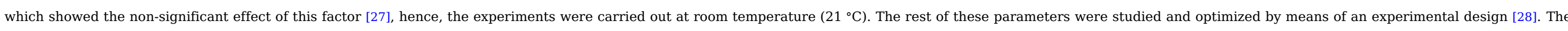

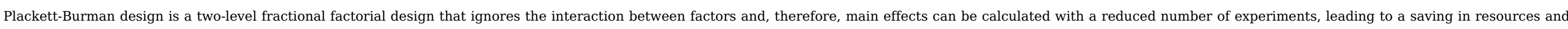

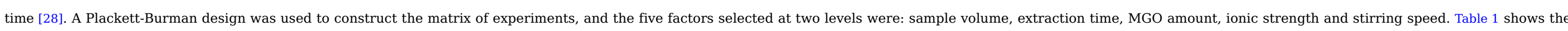

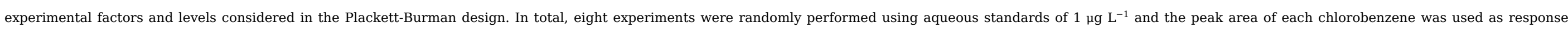
function.

Table 1 Experimental factors and levels of the Plackett-Burman design.

alt-text: Table 1

\begin{tabular}{|c|c|c|}
\hline \multirow[t]{2}{*}{ Factor } & \multicolumn{2}{|c|}{ Level } \\
\hline & Low & High \\
\hline Sample volume (mL) & 5 & 20 \\
\hline Extraction time (min) & 5 & 30 \\
\hline MGO amount (mg) & 1 & 10 \\
\hline Ionic strength ( $\mathrm{NaCl}$ concentration; \%, w/v) & 0 & 15 \\
\hline Stirring speed (rpm) & 500 & 1500 \\
\hline
\end{tabular}

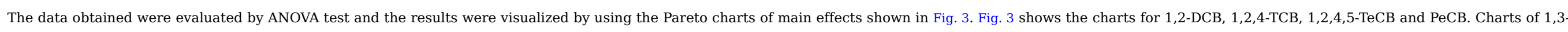

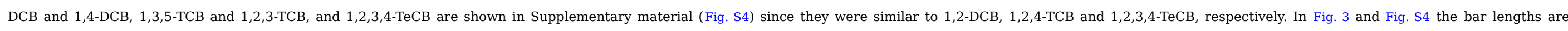

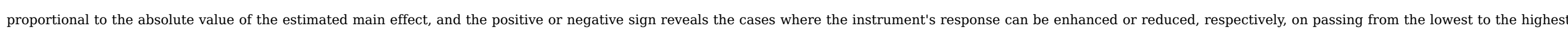

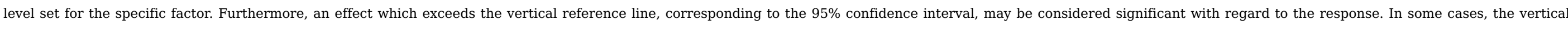

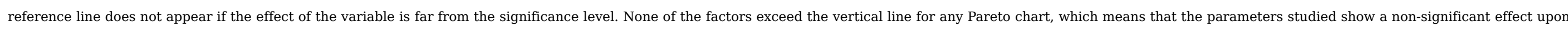



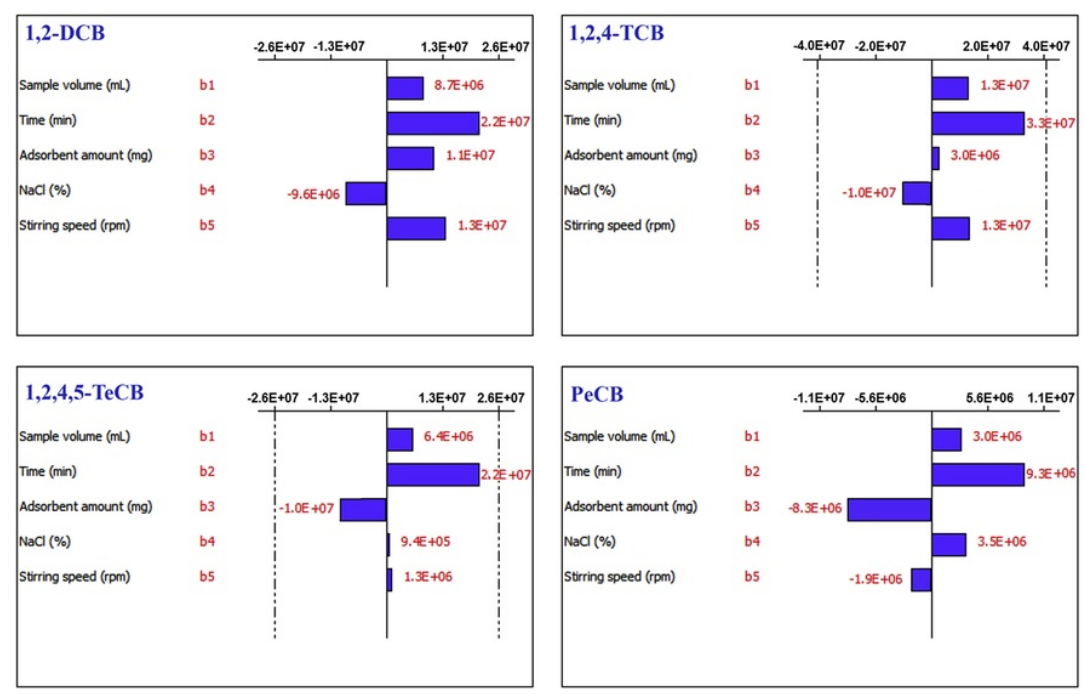

Fig. 3 Pareto charts of the main effects obtained from a Plackett-Burman design for 1,2-DCB, 1,2,4-TCB, 1,2,3,4-TeCB 1,2,4,5-TeCB and PeCB.

\section{alt-text: Fig. 3}

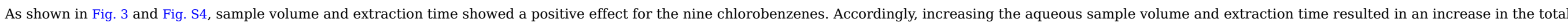

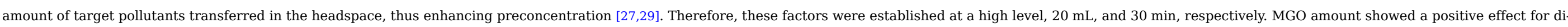

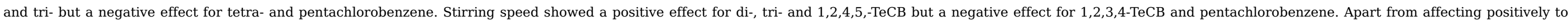

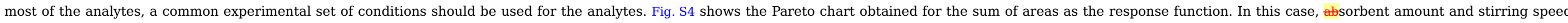

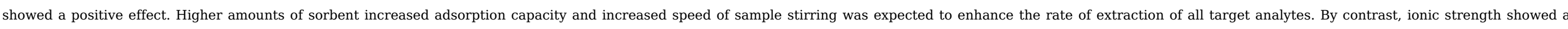

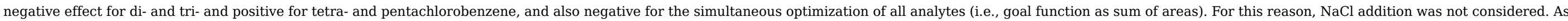

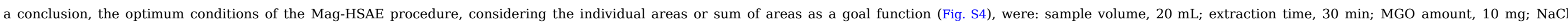
concentration, $0 \%$, and; stirring speed, $1500 \mathrm{rpm}$.

\subsection{Analytical parameters}

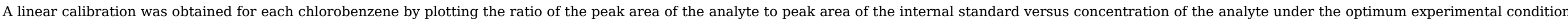
(Fig. S5). The calibration parameters, extracted from the calibration curves with determination coefficients $\left(\mathrm{R}^{2}\right)$ higher than 0.99 , are shown in Table 2.

Table 2 Analytical parameters of the investigated chlorobenzenes using the proposed Mag-HSAE-TD-GC-MS method.

alt-text: Table 2

Analyte

Dynamic range $\left(\mathrm{ng} \mathrm{L}^{-1}\right)^{\mathrm{a}}$

Slope (area units L ng-1)

CV $(\%)^{\mathrm{b}}$

$10 \mathrm{ng} \mathrm{L}^{-1}$

$50 \mathrm{ng} \mathrm{L^{-1 }}$

LOD (pg L-1)

LOQ $\left(p g \mathrm{~L}^{-1}\right)$

\begin{tabular}{|c|c|c|c|c|c|c|c|}
\hline 1,3-DCB & $0.5-100(6)$ & 0.1038 & 0.994 & 3.9 & 2.8 & 125 & 417 \\
\hline 1,4-DCB & $0.5-100(6)$ & 0.1266 & 0.992 & 3.3 & 3.2 & 93 & 310 \\
\hline
\end{tabular}




\begin{tabular}{|c|c|c|c|c|c|c|c|}
\hline 1,2-DCB & $0.5-100(6)$ & 0.0856 & 0.993 & 4.2 & 3.4 & 173 & 577 \\
\hline 1,3,5-ТСВ & $0.5-100(6)$ & 0.1048 & 0.994 & 4.5 & 1.5 & 193 & 643 \\
\hline 1,2,4-ТСВ & $0.5-100(6)$ & 0.0804 & 0.991 & 9.5 & 6.3 & 131 & 437 \\
\hline 1,2,3-ТСВ & $0.5-100(6)$ & 0.0547 & 0.992 & 8.9 & 4.2 & 153 & 510 \\
\hline $1,2,4,5-\mathrm{TeCB}$ & $0.5-75(5)$ & 0.0159 & 0.996 & 7.7 & 3.6 & 134 & 447 \\
\hline $1,2,3,4-\mathrm{TeCB}$ & $1-75(5)$ & 0.0018 & 0.993 & 8.7 & 7.5 & 301 & 1003 \\
\hline РеСВ & $0.5-75(5)$ & 0.0071 & 0.992 & 7.0 & 5.4 & 149 & 497 \\
\hline
\end{tabular}

a Number of calibration points in parentheses.

$\mathbf{b}_{\mathrm{n}}=5$.

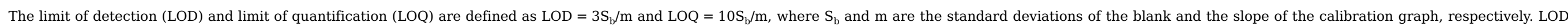

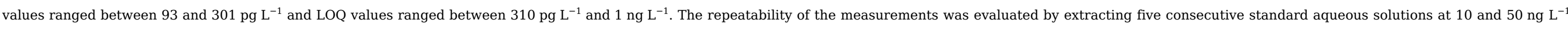

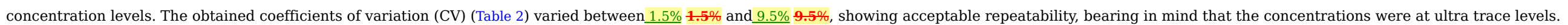

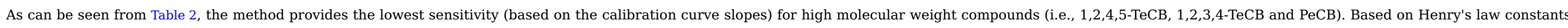

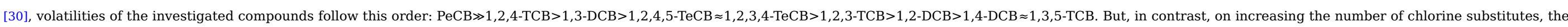

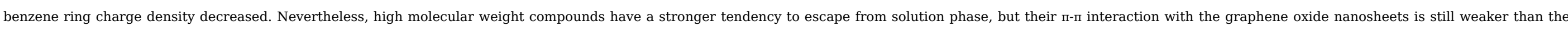

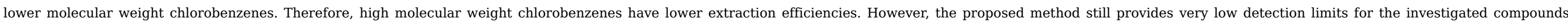
compared to previously reported works (Table 3).

Table 3 Comparison of the developed Mag-HSAE-TD-GC-MS method with some previously reported headspace extraction methods for analysis of chlorobenzenes in aqueous samples. alt-text: Table 3

\begin{tabular}{|c|c|c|c|c|c|c|c|c|c|c|c|c|c|c|c|}
\hline \multirow[t]{2}{*}{ Extraction method } & \multirow[t]{2}{*}{ Dynamic range (ng L-1) } & \multicolumn{9}{|c|}{ LOD $\left(\right.$ ng L L-1) $^{-1}$} & \multirow[t]{2}{*}{ CV (\%) } & \multirow[t]{2}{*}{ Extraction Temp. $\left({ }^{\circ} \mathrm{C}\right)$} & \multirow[t]{2}{*}{ Extraction time (min) } & \multirow[t]{2}{*}{ Detection method } & \multirow[t]{2}{*}{ Ref. } \\
\hline & & $\begin{array}{l}1,3- \\
\text { DCB }\end{array}$ & $\begin{array}{l}1,4- \\
\text { DCB }\end{array}$ & $\begin{array}{l}1,2- \\
\text { DCB }\end{array}$ & $\begin{array}{l}1,3,5- \\
\text { TCB }\end{array}$ & $\begin{array}{l}1,2,4- \\
\text { TCB }\end{array}$ & $\begin{array}{l}\text { 1,2,3- } \\
\text { TCB }\end{array}$ & $\begin{array}{c}\text { 1,2,4,5- } \\
\text { TeCB }\end{array}$ & $\begin{array}{c}\text { 1,2,3,4- } \\
\text { TeCB }\end{array}$ & РeCB & & & & & \\
\hline G-SPME & $\begin{array}{l}2500-500000 \\
2500-800000\end{array}$ & 800 & - & 500 & - & 500 & - & - & - & - & & 20 & 15 & GC-FID & [31] \\
\hline IL-SDME & $5000-160000$ & 152 & 203 & 102 & 122 & 122 & 102 & 102 & 102 & - & $1.6-5.1$ & RT & 37 & LC-UV & [27] \\
\hline Dodecane-HSME & $\begin{array}{l}500-3000 \\
5000-30000\end{array}$ & 100 & 3000 & 200 & - & - & - & - & - & - & $<10$ & 45 & 5 & GC-ECD & [32] \\
\hline PS-SPME & $\begin{array}{l}240-24000 \\
5-500 \\
0.5-20\end{array}$ & - & 75 & 40 & - & 1.5 & - & - & 0.15 & - & $2.1-9.7$ & 35 & 5 & GC-ECD & [33] \\
\hline MW-SDME & $1000-320000$ & 32 & 39 & 24 & 22 & 22 & 16 & 16 & 16 & - & $2.3-8.3$ & 21 & 20 & LC-UV & [29] \\
\hline PC-SPME & $\begin{array}{l}250-250000 \\
490-250000\end{array}$ & 31 & - & 62 & - & 62 & - & - & - & - & & 40 & 30 & GC-FID & [34] \\
\hline PU-SPME & $\begin{array}{l}50-900 \\
50-1000\end{array}$ & - & 10 & 10 & - & 10 & 10 & - & 10 & - & $3-11.3$ & 30 & 10 & GC-MS & [35] \\
\hline
\end{tabular}




\begin{tabular}{|c|c|c|c|c|c|c|c|c|c|c|c|c|c|c|c|}
\hline PDMS-SPME & $20-20000$ & 6 & 6 & 6 & 4 & 4 & 4 & 3 & 3 & 4 & $1.2-8.2$ & RT & 30 & GC-MS & [3] \\
\hline HSSE & $7-1000$ & 6 & 4 & 2 & 4 & 7 & 9 & 7 & 11 & 12 & $5-10$ & RT & 60 & GC-MS & [36] \\
\hline Toluene-SDME & $20-50000$ & 3 & 6 & 6 & 4 & 6 & 6 & 3 & 3 & 16 & $2.1-13.2$ & $\mathrm{RT}$ & 5 & GC-MS & [37] \\
\hline IL-SDME & $\begin{array}{l}20-2500 \\
20-5000 \\
20-10000 \\
20-15000\end{array}$ & 3 & 2 & 2 & 4 & 2 & 2 & 2 & 2 & 1 & $2-17$ & $\mathrm{RT}$ & 37 & GC-MS & [25] \\
\hline CA-SPME & $\begin{array}{l}8-800 \\
80-8000\end{array}$ & - & - & - & 2.25 & 1.64 & 0.94 & 1.26 & 0.32 & 0.50 & $2.1-4.9$ & 20 & 15 & GC-ECD & [38] \\
\hline PPy-SPME & $\begin{array}{l}5-1000 \\
2.5-1000\end{array}$ & - & 0.8 & 0.8 & - & 0.6 & - & - & 0.5 & - & $3-14$ & 65 & 25 & GC-MS & [39] \\
\hline MOF/PANI-SPME & $0.5-1000$ & - & 0.2 & 0.1 & - & 0.1 & 0.1 & - & 0.1 & - & $5-8$ & 25 & 20 & GC-MS & [40] \\
\hline Mag-HSAE & $\begin{array}{l}0.5-100 \\
0.5-75\end{array}$ & 0.12 & 0.09 & 0.17 & 0.19 & 0.13 & 0.15 & 0.13 & 0.30 & 0.15 & $1.5-9.5$ & $\mathrm{RT}$ & 30 & GC-MS & $\begin{array}{l}\text { This } \\
\text { work }\end{array}$ \\
\hline
\end{tabular}

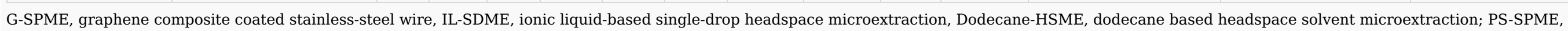

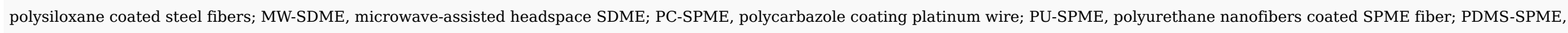

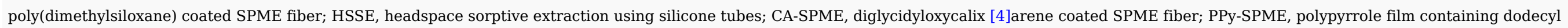

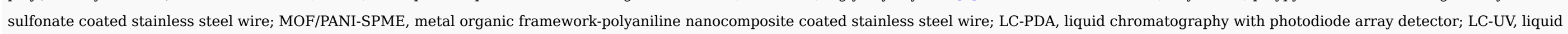

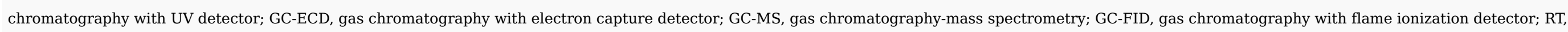
room temperature.

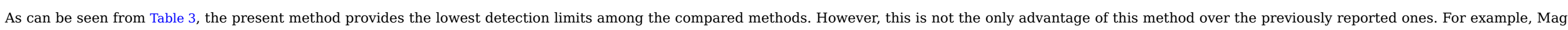

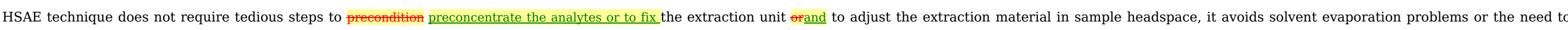

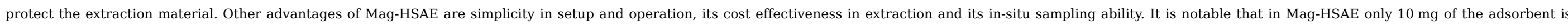
needed for each experiment, and due to the MGO, relatively cheap precursors and simple synthesis procedure it is very cost effective.

\subsection{Real water samples}

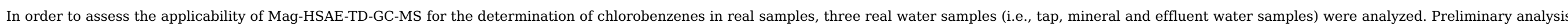

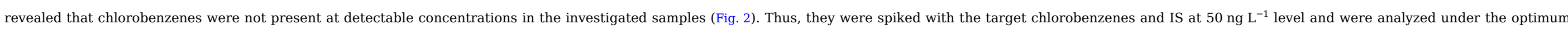

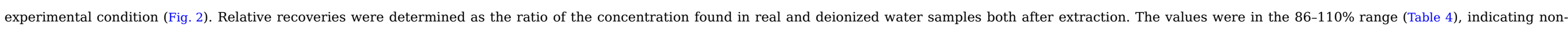
significant matrix effects on the extraction performance of the Mag-HSAE method for extraction of the investigated chlorobenzenes.

Table 4 Real water samples analysis by the Mag-HSAE-TD-GC-MS method.

alt-text: Table 4

Analyte

Tap water
Relative recoveries ${ }^{\mathrm{a}}(\%)$ and $\mathrm{CV}$ in parenthesis (\%)

Mineral water

Effluent water

\begin{tabular}{|l|l|l|l|}
\hline $1,3-\mathrm{DCB}$ & $103(2.1)$ & $108(2.8)$ & $98(3.6)$ \\
\hline $1,4-\mathrm{DCB}$ & $97(4.3)$ & $105(3.2)$ & $101(4.5)$ \\
\hline
\end{tabular}




\begin{tabular}{|l|l|l|l|l|}
\hline $1,2-\mathrm{DCB}$ & $110(2.6)$ & $104(3.4)$ & $95(3.7)$ \\
\hline $1,3,5-\mathrm{TCB}$ & $95(4.5)$ & $101(1.5)$ & $94(6.3)$ & $103(2.6)$ \\
\hline $1,2,4-\mathrm{TCB}$ & $109(3.4)$ & $108(4.2)$ & $96(5.3)$ \\
\hline $1,2,3-\mathrm{TCB}$ & $105(2.8)$ & $98(3.6)$ & $96(7.2)$ \\
\hline $1,2,4,5-\mathrm{TeCB}$ & $97(5.3)$ & $102(7.5)$ & $87(6.5)$ \\
\hline $1,2,3,4-\mathrm{TeCB}$ & $95(5.2)$ & $100(5.4)$ & $90(4.3)$ \\
\hline РеСВ & $103(8.1)$ & $105(4.4)$ \\
\hline
\end{tabular}

a Mean value of three extractions at $50 \mathrm{ng} \mathrm{L}^{-1}$ concentration level.

\section{Conclusions}

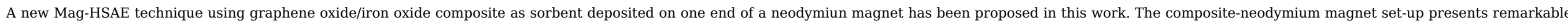

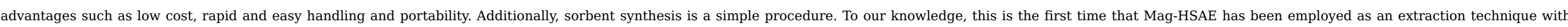

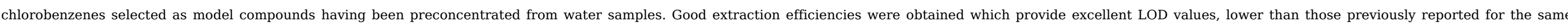

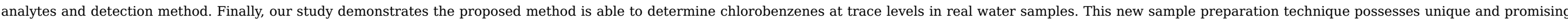

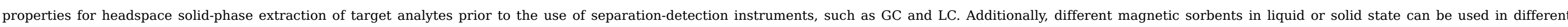
situations for simple, fast, sensitive and cost effective headspace extraction of a wide range of analytes from different matrixes.

\section{Acknowledgements}

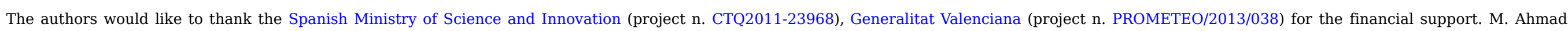
would like to thank Iranian Ministry of Science, Research, and Technology for the travel grant. E. Fernández thanks Spanish Ministry of Education for her FPU grant (FPU13/03125).

\section{Appendix A. Supplementary data}

Supplementary data related to this article can be found at http://dx.doi.org/10.1016/j.aca.2017.04.002.

\section{References}

[1] J. Pawliszyn, Theory of extraction, In: J. Pawliszyn and H.L. Lord, (Eds.), Handbook of Sample Preparation, 2010, John Wiley \& Sons, Inc, 1-24.

[2] F. Pena-Pereira, Miniaturization in Sample Preparation, 2014, Walter de Gruyter GmbH \& Co KG; Berlin

[3] Y. He, Y. Wang and H.K. Lee, Trace analysis of ten chlorinated benzenes in water by headspace solid-phase microextraction, J. Chromatogr. A 874, $2000,149-154$.

[4] S. Van Bramer and K.R. Goodrich, Determination of plant volatiles using solid phase microextraction GC-MS, J. Chem. Educ. 92, 2015, 916-919.

[5] J.W. Zewe, J.K. Steach and S.V. Olesik, Electrospun fibers for solid-phase microextraction, Anal. Chem. 82, 2010, 5341-5348.

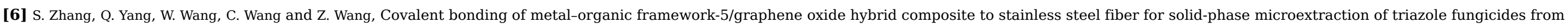
fruit and vegetable samples, J. Agri. Food Chem. 64, 2016, 2792-2801.

[7] C.L. Arthur and J. Pawliszyn, Solid phase microextraction with thermal desorption using fused silica optical fibers, Anal. Chem. 62, 1990, 2145-2148.

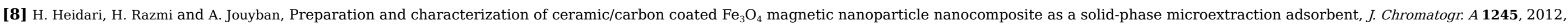
$1-7$. 
[9] M. de Fátima Alpendurada, Solid-phase microextraction: a promising technique for sample preparation in environmental analysis, J. Chromatogr. A 889, 2000 , 3-14.

[10] Z. Zhang and J. Pawliszyn, Headspace solid-phase microextraction, Anal. Chem. 65, 1993, 1843-1852.

[11] B. Tienpont, F. David, C. Bicchi and P. Sandra, High capacity headspace sorptive extraction, J. Microcolumn Sep. 12, $2000,577-584$.

[12] J. Płotka-Wasylka, N. Szczepańska, M. de la Guardia and J. Namieśnik, Miniaturized solid-phase extraction techniques, TrAC-Trends Anal. Chem. 73, $2015,19-38$.

[13] S. Tang, H. Zhang and H.K. Lee, Advances in sample extraction, Anal. Chem. 88, 2016, 228-249.

[14] A. Cappiello, A. Berloni, G. Famiglini, F. Mangani and P. Palma, Micro-SPE method for sample introduction in capillary HPLC/MS, Anal. Chem. 73, $2001,298-302$.

[15] X. Huang, L. Chen, F. Lin and D. Yuan, Novel extraction approach for liquid samples: stir cake sorptive extraction using monolith, J. Sep. Sci. 34, 2011 , $2145-2151$.

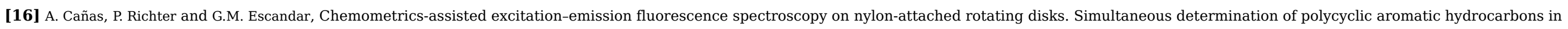
the presence of interferences, Anal. Chim. Acta 852, 2014, 105-111.

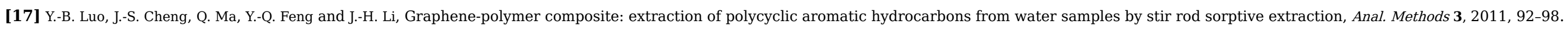

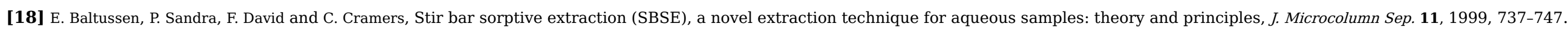

[19] M. Abdel-Rehim, Microextraction by packed sorbent (MEPS): a tutorial, Anal. Chim. Acta 701, 2011, 119-128.

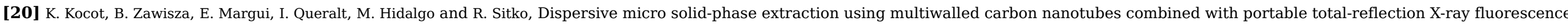
spectrometry for the determination of trace amounts of $\mathrm{Pb}$ and $\mathrm{Cd}$ in water samples, J. Anal. At. Spectrom. 28, 2013, 736-742.

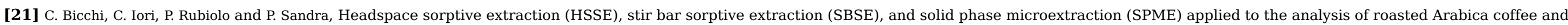
coffee brew, J. Agri. Food Chem. 50, 2002, 449-459.

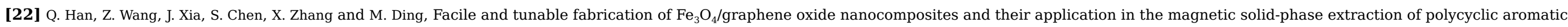
hydrocarbons from environmental water samples, Talanta 101, 2012, 388-395.

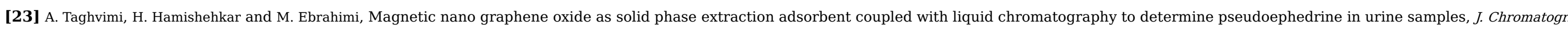
B 1009-1010, 2016, 66-72.

[24] Y. Zhu, S. Murali, W. Cai, X. Li, J.W. Suk, J.R. Potts and R.S. Ruoff, Graphene and graphene oxide: synthesis, properties, and applications, Adv. Mater 22, $2010,3906-3924$.

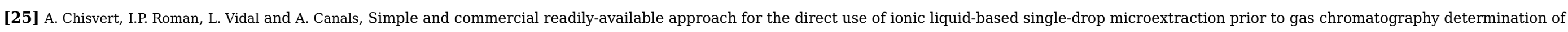
chlorobenzenes in real water samples as model analytical application, J. Chromatogr. A 1216, 2009, 1290-1295.

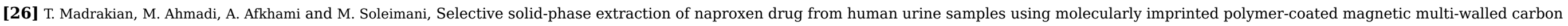
nanotubes prior to its spectrofluorometric determination, Analyst 138, 2013, 4542-4549.

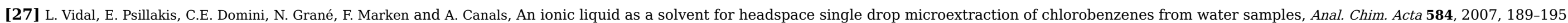

[28] D.C. Montgomery, Design and Analysis of Experiments, seventh ed., 2009, John Wiley \& Sons, Inc.; New Jersey, USA.

[29] L. Vidal, C.E. Domini, N. Grane, E. Psillakis and A. Canals, Microwave-assisted headspace single-drop microextration of chlorobenzenes from water samples, Anal. Chim. Acta 592, 2007 , 9-15.

[30] D. Mackay and W.Y. Shiu, A critical review of Henry's law constants for chemicals of environmental interest, J. Phys. Chem. Ref. Data 10, 1981, 1175-1199.

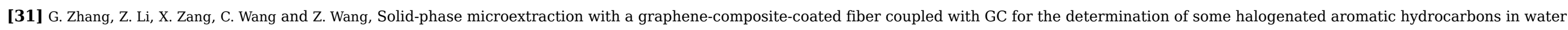
samples, J. Sep. Sci. 37, 2014, 440-446. 


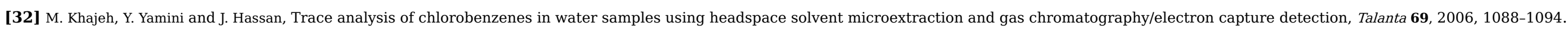

[33] M. Saraji and N. Mehrafza, Polysiloxane coated steel fibers for solid-phase microextraction of chlorobenzenes, Microchim. Acta 182, 2015, 841-848.

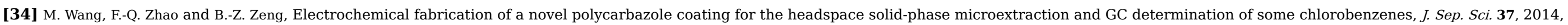
861-867.

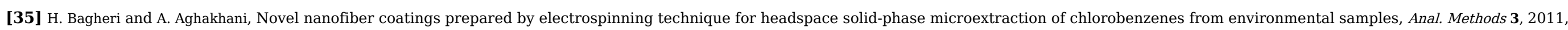
$1284-1289$.

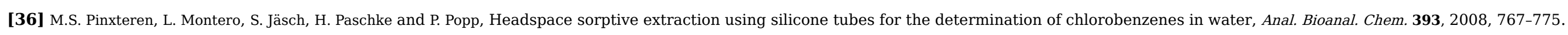

[37] L. Vidal, A. Canals, N. Kalogerakis and E. Psillakis, Headspace single-drop microextraction for the analysis of chlorobenzenes in water samples, J. Chromatogr. A 1089, 2005, 25-30.

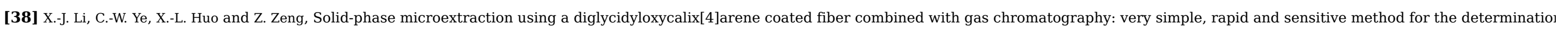
of chlorobenzenes in water, Microchim. Acta 168, 2010, 161-167.

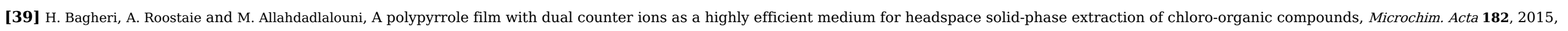
617-624.

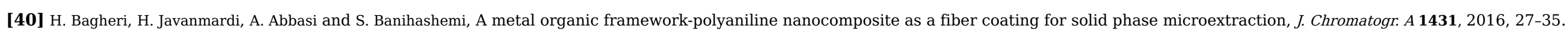

\section{Appendix A. Supplementary data}

The following is the supplementary data related to this article:

Multimedia Component 1

Supplementary material

alt-text: Supplementary material

Graphical abstract

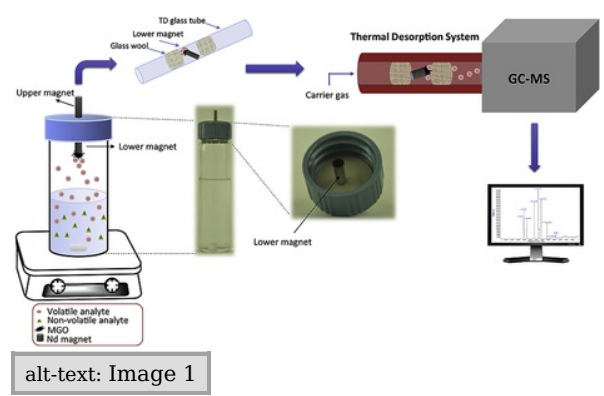

Highlights 
- Graphene oxide/iron oxide composite deposited on a neodymiun magnet as sorbent.

- Sorbent of low cost, rapid and simple synthesis, easy manipulation and portability options.

- Fast and efficient extraction and sensitive determination of chlorobenzenes in water samples.

\section{Queries and Answers}

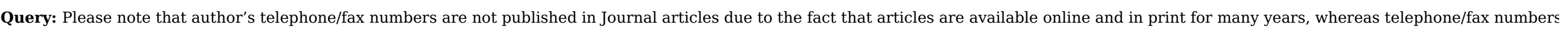
are changeable and therefore not reliable in the long term.

Answer: Ok, not problem at all.

Query: Could you please provide the grant number for Iranian Ministry of Science, Research, and Technology, if any?

Answer: There is no grant number for this case.

Query: Please confirm that given names and surnames have been identified correctly.

Answer: Yes

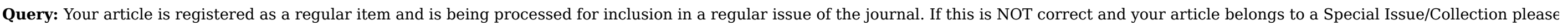
contact a.hubertt@elsevier.com immediately prior to returning your corrections.

Answer: This article does not belong to a Special Issue. 